\title{
Papel da glucosamina no alívio dos sintomas da osteoartrose: revisão baseada na evidência
}

Liliana Ferreira, ${ }^{1-2}$ Eva Gomes ${ }^{2-3}$

\section{RESUMO}

Objetivo: A osteoartrose (OA) é a principal causa de dor e incapacidade física em idosos. A glucosamina tem atraído grande interesse como fármaco específico na OA, quer para o alívio da dor quer para a melhoria da função articular, parecendo ser segura. Contudo, muitos estudos têm demonstrado diferentes níveis de eficácia, pelo que o seu uso permanece controverso. Assim, o objetivo deste trabalho foi rever a evidência disponível sobre a eficácia e segurança da glucosamina no alívio dos sintomas da OA.

Fontes de dados: Cochrane Library, PubMed, National Guideline Clearinghouse, DARE, TRIP Database, Bandolier, Index Revistas Médicas Portuguesas.

Métodos de revisão: Pesquisa de meta-análises (MA), revisões sistemáticas, ensaios clínicos aleatorizados e controlados (ECAC) e normas de orientação clínica (NOC), publicados entre 01/2009 e 12/2012, utilizando os termos MeSH osteoarthritis e glucosamine e correspondentes DeCS. Para atribuição dos níveis de evidência e forças de recomendação foi usada a escala Strenght of Recommendation Taxonomy (SORT), da American Family Physician.

Resultados: Obtiveram-se 200 artigos, dos quais quatro cumpriam os critérios de inclusão: duas MA, um ECAC e uma NOC, perfazendo um total de 9.428 indivíduos estudados. Os três artigos demonstraram que o uso da glucosamina tem algum benefício no alívio da dor e na melhoria da função articular, mas que este benefício não é clinica nem estatisticamente significativo comparativamente ao placebo. Contudo, a glucosamina mostrou-se tão segura quanto o placebo. A NOC não recomenda o uso da glucosamina na OA.

Conclusão: A evidência atual disponível indica que o benefício da glucosamina não é clinicamente significativo no alívio dos sintomas da OA, nomeadamente no alívio da dor e na melhoria da função articular (SOR A). Contudo, o seu uso é seguro (SOR A). Assim, a realização de estudos futuros que avaliem o custo/benefício da glucosamina no alívio dos sintomas constituirá uma mais-valia para encorajar ou desencorajar o seu uso, principalmente quando esta terapêutica é comparticipada pelo Serviço Nacional de Saúde.

Palavras-chave: Glucosamina; Osteoartrose; Artralgia.

\section{INTRODUÇÃO}

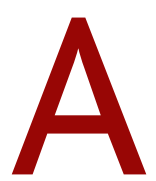
osteoartrose (OA) é uma doença articular degenerativa progressiva, com sinais inflamatórios mínimos e cujas manifestações clínicas incluem anomalias estruturais e um conjunto de sintomas caracterizados por dor e limitação funcional, com consequente redução da qualidade de vida.

Os fatores de risco para o seu desenvolvimento in-

\footnotetext{
${ }^{1}$ Unidade de Saúde Familiar Novo Sentido - ACES Grande Porto VI - Porto Oriental.

${ }^{2}$ Médica interna de Medicina Geral e Familiar.

${ }^{3}$ Unidade de Saúde Familiar Nova Via - ACES Grande Porto VIII - Espinho/Gaia.
}

cluem a idade, sexo feminino, obesidade, sobrecarga articular excessiva, trauma, lesões periarticulares e alguns riscos ocupacionais. ${ }^{2-3}$ Alguns autores consideram ainda a presença de componentes genéticos. ${ }^{3}$

A Organização Mundial de Saúde descreve a OA como a quarta principal causa de incapacidade nas mulheres e a oitava entre os homens, ${ }^{3}$ constituindo a principal causa de dor e incapacidade física em idosos. ${ }^{4-5}$

A sua prevalência aumenta com a idade, de aproximadamente $7,0 \%$ nos indivíduos entre os 65 e os 70 anos a $11,2 \%$ entre aqueles com idade igual ou superior a 80 anos. $^{6-7}$ 
Existem vários instrumentos capazes de medir diferentes dimensões do estado de saúde dos indivíduos com OA. Entre estes, há dois amplamente utilizados: o Western Ontario and McMaster Universities Osteoarthritis Index (WOMAC) ${ }^{8-9} \mathrm{e} 0$ índice de Lequesne. ${ }^{10} \mathrm{Ambos}$ avaliam sintomas e incapacidade física. ${ }^{11}$

No que respeita ao tratamento, a OA é um problema de saúde pública para o qual há poucas terapêuticas eficazes. ${ }^{12} \mathrm{O}$ alívio da dor é, de facto, dominado pelo uso de analgésicos, anti-inflamatórios não esteróides (AINE), inibidores seletivos da cicloxigenase-2 (COX-2) e administrações intra-articulares de ácido hialurónico ou corticosteróides. No entanto, existem dados consideráveis relativos ao benefício reduzido destas terapêuticas, com efeitos secundários não desprezíveis de alguns deles, ${ }^{13-16}$ pelo que não é surpreendente a procura de abordagens alternativas para o controlo dos sintomas da AO. ${ }^{17-21}$

A glucosamina tem atraído um grande interesse como um fármaco específico para a $\mathrm{AO},{ }^{22}$ parecendo ser segura. ${ }^{23}$ Não obstante, muitos estudos têm demonstrado vários níveis de eficácia do uso da glucosamina em doentes com $\mathrm{AO}^{24}$

Com base em dados publicados, em 2003, a European League Against Rheumatism (EULAR) ${ }^{3,25} \mathrm{e}$, em 2007, a Osteoarthritis Research Society International (OARSI), ${ }^{26-27}$ passaram a recomendar o uso de sulfato de glucosamina como terapêutica da OA da anca e do joelho, embora esta última desaconselhasse a continuidade do mesmo quando após 6 meses de tratamento não se evidenciasse uma melhoria da sintomatologia. ${ }^{24}$

No entanto, mais recentemente observou-se uma alteração na evidência relatada pela OARSI, após uma pesquisa cumulativa sistemática realizada até janeiro de $2009 .{ }^{28}$ Esta meta-análise relata uma progressiva diminuição da eficácia da glucosamina, nomeadamente no controlo da dor, quando a evidência dos estudos controlados aleatorizados era cronologicamente avaliada, revelando a heterogeneidade de resultados e a presença do viés de publicação, ${ }^{24} \mathrm{o}$ que veio alterar a recomendação da OARSI, que passou a preconizar que o uso da glucosamina no alívio dos sintomas da OA deva ser ponderado caso a caso pelo médico, tendo em conta a evidência limitada da sua eficácia. ${ }^{28}$

Com efeito, o uso de glucosamina na abordagem terapêutica da OA permanece controverso e o seu meca- nismo de ação específico no controlo da dor e na melhoria da função articular permanecem incertos. ${ }^{24}$

Assim, o objetivo deste trabalho foi rever a evidência disponível sobre o uso da glucosamina em doentes com OA, no que respeita à eficácia no alívio da dor e melhoria da função articular, bem como à segurança relativamente aos efeitos secundários, quando comparada com o placebo.

\section{MÉTODOS}

Foi realizada uma pesquisa bibliográfica em janeiro de 2013, em duplicado pelas duas autoras (com uma taxa de concordância de $100 \%$ ), utilizando os termos MeSH osteoarthritis e glucosamine. Pesquisaram-se meta-análises (MA), revisões sistemáticas, ensaios clínicos aleatorizados e controlados (ECAC) e normas de orientação clínica (NOC) nas bases de dados da Cochrane Library, PubMed, National Guideline Clearinghouse, DARE, TRIP Database, Bandolier, Index Revistas Médicas Portuguesas, publicadas entre janeiro de 2009 e dezembro de 2012, em inglês e português (línguas dominadas pelas autoras). Procedeu-se à análise de referências cruzadas dos artigos. Para a atribuição dos níveis de evidência e forças de recomendação foi usada a escala Strenght of Recommendation Taxonomy (SORT), da American Family Physician..$^{29}$

A população em estudo incluiu todos os adultos com idade igual ou superior a 18 anos com diagnóstico de OA primária ou secundária em qualquer articulação, incluindo o esqueleto axial e periférico. Foi excluída a população em idade pediátrica devido à incidência muito baixa desta patologia neste grupo etário. A intervenção avaliada consistiu no uso de glucosamina em comparação com o placebo. Como outcomes/resultados de eficácia definiram-se o alívio da dor e a melhoria da função articular; como outcome/resultado de segurança definiu-se a incidência de efeitos adversos.

\section{RESULTADOS}

Foram encontrados 200 artigos, dos quais quatro cumpriam os critérios de inclusão: duas MA, um ECAC e uma NOC, perfazendo um total de 9.428 indivíduos estudados. Os restantes artigos foram excluídos por não cumprirem os critérios de inclusão, por divergirem do objetivo do trabalho ou por serem artigos repetidos ou estarem incluídos nas MA. 


\begin{tabular}{|c|c|c|c|c|c|}
\hline & Referência & $\begin{array}{l}\text { População/ } \\
\text { Intervenção }\end{array}$ & $\begin{array}{l}\text { Outcomes/ } \\
\text { Resultados }\end{array}$ & Conclusões & NE \\
\hline MA & $\begin{array}{l}\text { Wandel et al } \\
(2010)^{30}\end{array}$ & $\begin{array}{l}10 \text { ECAC } \\
\mathrm{n}=3.803 \\
\text { doentes } \\
\text { Glucosamina e } \\
\text { condroitina } \\
\text { isoladamente } \\
\text { ou em associação } \\
\text { vs placebo }\end{array}$ & $\begin{array}{l}\text { Alívio da dor; } \\
\text { Efeitos adversos }\end{array}$ & $\begin{array}{l}\text { A glucosamina e a condroitina, isoladamente ou em } \\
\text { associação, não têm eficácia clinicamente relevante no } \\
\text { alívio da dor, embora em alguns estudos a diminuição } \\
\text { da intensidade da dor se tenha mostrado estatis- } \\
\text { ticamente significativa. } \\
\text { Os ensaios clínicos independentes das indústrias } \\
\text { farmacêuticas demonstraram menor eficácia que os } \\
\text { financiados por estas últimas. } \\
\text { Sem impacto sobre o estreitamento do espaço articular. } \\
\text { O seu uso é seguro. }\end{array}$ & 1 \\
\hline MA & $\begin{array}{l}\text { Towheed et } \\
\text { al. Cochrane } \\
\text { Review } \\
(2009)^{31}\end{array}$ & $\begin{array}{l}25 \text { ECAC } \\
\mathrm{n}=4.963 \\
\text { doentes com } \\
\text { idade } \geq 18 \text { anos } \\
\text { Glucosamina vs } \\
\text { placebo }\end{array}$ & $\begin{array}{l}\text { Alívio da dor; } \\
\text { Melhoria da } \\
\text { função articular; } \\
\text { Efeitos adversos }\end{array}$ & $\begin{array}{l}\text { Não existe qualquer benefício da glucosamina no alívio } \\
\text { da dor, avaliada por diversas escalas, incluindo a escala } \\
\text { WOMAC, nem na função articular. Contudo, verificou-se } \\
\text { uma eficácia superior da glucosamina em relação ao } \\
\text { placebo quando utilizado o índice de Lequesne, embora } \\
\text { os resultados não tenham sido uniformemente positivos } \\
\text { nem estatisticamente significativos. } \\
\text { A eficácia moderada da glucosamina no alívio da dor e } \\
\text { na função articular está na dependência dos resultados } \\
\text { dos ensaios clínicos realizados com sulfato de } \\
\text { glucosamina. } \\
\text { A glucosamina mostrou-se tão segura quanto o placebo. }\end{array}$ & 2 \\
\hline ECAC & $\begin{array}{l}\text { Sawitzke et } \\
\text { al }(2010)^{32}\end{array}$ & $\begin{array}{l}\mathrm{n}=662 \text { com } \\
\text { idade } \geq 40 \text { anos } \\
\text { Glucosamina e } \\
\text { condroitina, } \\
\text { isoladamente ou } \\
\text { em associação, } \\
\text { vs placebo }\end{array}$ & $\begin{array}{l}\text { Alívio da dor; } \\
\text { Melhoria da } \\
\text { função articular; } \\
\text { Efeitos adversos }\end{array}$ & $\begin{array}{l}\text { Passados dois anos, nenhum tratamento alcançou } \\
\text { diferenças clinicamente importantes na dor ou na } \\
\text { função articular, com base na escala WOMAC, quando } \\
\text { comparado com o placebo, apesar de se observar algum } \\
\text { benefício com o uso de glucosamina. } \\
\text { Registaram-se efeitos adversos semelhantes entre os } \\
\text { diversos tratamentos. }\end{array}$ & 1 \\
\hline
\end{tabular}

Legenda: MA - Meta-análise; ECAC - Ensaio clínico aleatorizado e controlado; OA - Osteoartrose; WOMAC - Western Ontario and McMaster Universities Osteoarthritis Index; NE - Nível de evidência; NE 1 - estudo de boa qualidade, evidência orientada para o doente; NE 2 - estudo de qualidade limitada, evidência orientada para o doente.

A descrição dos artigos incluídos encontra-se resumida no Quadro I.

A MA de Wandel et al,,$^{30}$ publicada em 2010 (NE 1), teve como objetivo principal determinar a eficácia do uso da glucosamina e da condroitina, isoladamente ou em associação, no alívio da dor na OA da anca ou do joelho. Os autores avaliaram 10 ECAC, todos com amostras superiores a 200 doentes com OA da anca ou do joe- lho, perfazendo um total de 3.803 doentes. Contudo, apenas 6 destes ECAC compararam a glucosamina com o placebo, perfazendo 1.309 doentes; houve ainda um braço de um sétimo estudo que também fez esta mesma comparação, totalizando, assim, 7 estudos, perfazendo uma amostra total de 1.939 doentes. A intensidade da dor constituiu o outcome primário. Os autores concluíram que, comparativamente ao placebo, a glu- 
cosamina não tem eficácia clinicamente relevante no alívio da dor, embora em alguns estudos a diminuição da intensidade da dor se tenha mostrado estatisticamente significativa. Os autores salientam ainda que os ensaios clínicos independentes das indústrias farmacêuticas demonstraram menor eficácia que os financiados por estas últimas. Não obstante a ausência de eficácia clinicamente relevante, atendendo à segurança que estes suplementos têm vindo a mostrar, os autores afirmam não haver contraindicação à sua utilização pelos doentes enquanto estes os considerarem benéficos e o seu custo seja suportado pelos próprios. Contudo, ressalvam que estes não deverão ser financiados pelos sistemas de saúde, uma vez que não existe evidência científica que encoraje o seu uso e que novas prescrições destes suplementos a doentes que não receberam tratamento prévio devem ser desencorajadas.

A mais recente MA da Cochrane, de Towheed et al, ${ }^{31}$ publicada em 2009 (NE 2), teve como principal objetivo avaliar a eficácia, sintomática e estrutural, e a segurança da glucosamina no tratamento farmacológico da OA. Esta revisão incluiu 25 estudos, perfazendo um total de 4.963 doentes com idade igual ou superior a 18 anos e diagnóstico primário ou secundário de $\mathrm{OA}$ (22 dos quais avaliaram apenas a OA do joelho e/ou anca e apenas 3 avaliaram a OA em qualquer articulação). Uma análise restrita aos estudos com adequada ocultação de alocação não conseguiu mostrar qualquer benefício da glucosamina no alívio da dor, avaliada por diversas escalas, incluindo a escala WOMAC, nem na função articular. Contudo, verificou-se uma eficácia superior da glucosamina em relação ao placebo quando utilizado o índice de Lequesne (desvio padrão (DP) -0,54; intervalo de confiança (IC) de 95\% [-0,96 a -0,12]). Globalmente, os 25 ECAC favoreceram o uso de glucosamina, observandose $22 \%$ de benefício no alívio da dor relativamente à linha de base (DP -0,47; IC de 95\% -0,72 a -0,23), bem como uma melhoria de $11 \%$ na função articular usando o índice de Lequesne (DP -0,47; IC de 95\% -0,82 a -0,12). No entanto, os resultados não foram uniformemente positivos nem estatisticamente significativos. De facto, apesar da análise global dos ensaios clínicos controlados por placebo evidenciar moderada eficácia da glucosamina no alívio da dor e na função articular, estes mostram grande heterogeneidade, nomeadamente no que se refere ao desenho e à qualidade dos mesmos, à prepara- ção da glucosamina e à dose utilizada. É importante referir ainda que a eficácia moderada da glucosamina no alívio da dor e na função articular está na dependência dos resultados dos ensaios clínicos realizados com sulfato de glucosamina, dado que outras preparações não mostraram qualquer efeito no alívio dos sintomas da OA. Os autores deste estudo referem ainda que os estudos que usaram preparações do laboratório Rotta demonstraram que a glucosamina tinha efeitos positivos em termos de dor e função articular, ao contrário dos estudos que usaram preparados não-Rotta de glucosamina que não demonstraram benefício deste fármaco em termos de dor ou função articular. Quanto à segurança da glucosamina, esta mostrou-se tão segura quanto o placebo.

O ECAC duplamente cego de Sawitzke et al, ${ }^{32}$ publicado em 2010 (NE 1), foi desenhado com o objetivo de avaliar a eficácia e segurança dos suplementos de glucosamina e sulfato de condroitina, isoladamente ou em combinação, comparativamente ao placebo, no alívio da dor nos doentes com OA do joelho. Uma amostra de 662 doentes, com pelo menos 40 anos de idade e evidência clínica (dor há pelo menos 6 meses) e radiológica de OA do joelho, foi aleatorizada em cinco grupos: ao grupo 1 foi administrada glucosamina, na dose de $500 \mathrm{mg}$, três vezes por dia; ao grupo 2 foi administrado sulfato de condroitina (SC), na dose de $400 \mathrm{mg}$, três vezes por dia; ao grupo 3 foi administrada a associação de glucosamina mais SC; ao grupo 4 foi administrada uma dose diária única de 200mg de celecoxib e ao grupo 5 foi administrado um placebo durante um período de 24 meses. Os autores definiram como outcome/resultado primário a redução de $20 \%$ da dor, com base na escala WOMAC, ao longo dos 24 meses e, como outcomes/resultados secundários, a melhoria da dor e da função articular com base nos critérios de OARSI. Passados dois anos, nenhum tratamento alcançou diferenças clinicamente importantes na dor ou na função articular, com base na escalaWOMAC, quando comparado com o placebo, apesar de se observar algum benefício com o uso de glucosamina, principalmente no que se refere aos outcomes/resultados secundários do estudo. Registaram-se efeitos adversos semelhantes entre os diversos tratamentos, com raros efeitos adversos graves.

A NOC do Colégio Americano de Reumatologia, publicada em 2012, ${ }^{33}$ recomenda "condicionalmente" que nos doentes com OA do joelho e/ou da anca não seja 
usada a glucosamina. Não há qualquer referência ao uso da glucosamina noutro tipo de OA.

\section{CONCLUSÃO}

Relativamente à eficácia, a evidência atual disponível indica que o benefício da glucosamina no alívio dos sintomas da OA, nomeadamente no alívio da dor e na melhoria da função articular, não é clínica nem estatisticamente significativo comparativamente ao placebo (SOR A).

No que respeita à segurança, a glucosamina mostrou-se tão segura quanto o placebo relativamente à ocorrência de efeitos adversos (SOR A).

O facto de os estudos serem globalmente heterogéneos, nomeadamente no que se refere ao desenho e à qualidade dos mesmos, à preparação da glucosamina e à dose, constituiu uma das principais limitações desta revisão. Outra das limitações foi o facto dos ensaios clínicos dependentes das indústrias farmacêuticas demonstrarem uma eficácia consistentemente superior relativamente aos ensaios clínicos independentes das indústrias farmacêuticas, adivinhando-se, por isso, um viés de publicação. Por último, uma vez que era objetivo desta revisão avaliar o benefício da glucosamina no alívio sintomático e melhoria da função articular na OA de qualquer articulação do esqueleto axial e periférico, o facto da maioria dos estudos avaliarem apenas o seu benefício na OA da anca e/ou joelho também constituiu uma limitação.

Futuramente será imprescindível a realização de mais estudos, independentes das indústrias farmacêuticas, com amostras de grandes dimensões, homogéneos e de boa qualidade, que avaliem o custo/benefício da glucosamina no alívio dos sintomas da OA, com o intuito de encorajar ou desencorajar o seu uso. Com efeito, tal necessidade requer particular atenção se tivermos presente que esta terapêutica é comparticipada pelo Sistema Nacional de Saúde, cujas normas de orientação defendem que para a mesma/semelhante eficácia e segurança, na ausência de contraindicações, a nossa linha de prescrição seja a do menor custo. Neste sentido, futuros estudos de análise de custo-benefício poderiam revelar-se uma mais-valia nesta matéria.

\section{REFERÊNCIAS BIBLIOGRÁFICAS}

1. Rovati LC, Girolami F, Persiani S. Crystalline glucosamine sulfate in the management of knee osteoarthritis: efficacy, safety, and pharmacoki- netic properties. Ther Adv Musculoskelet Dis. 2012;4(3):167-80.

2. Dawson J, Juszczak E, Thorogood M, Marks SA, Dodd C, Fitzpatrick R. An investigation of risk factors for symptomatic osteoarthritis of the Knee in women using a life course approach. J Epidemiol Community Health. 2003;57(10):823-30.

3. Jordan KM, Arden NK, Doherty M, Bannwarth B, Bijlsma JW, Dieppe P, et al. EULAR recommendations 2003: an evidence based approach to the management of knee osteoarthritis: Report of a Task Force of the Standing Committee for International Clinical Studies Including Therapeutic Trials (ESCISIT). Ann Rheum Dis. 2003;62(12):1145-55.

4. Rabenda V, Manette C, Lemmens R, Mariani AM, Struvay N, Reginster JY. Prevalence and impact of osteoarthritis and osteoporosis on healthrelated quality of life among active subjects. Aging Clin Exp Res. 2007;19(1):55-60.

5. Jinks $C$, Jordan $\mathrm{K}$, Croft P. Osteoarthritis as a public health problem: the impact of developing knee pain on physical function in adults living in the community (KNEST 3). Rheumatology (Oxford). 2007;46(5):87781.

6. Faucher M, Poiraudeau S, Lefevre-Colau MM, Rannou F, Fermanian J, Revel M. Algo-functional assessment of knee osteoarthritis: comparison of the test-retest reliability and construct validity of the WOMAC and Lequesne indexes. Osteoarthritis Cartilage. 2002;10(8):602-10.

7. Faucher M, Poiraudeau S, Lefevre-Colau MM, Rannou F, Fermanian J, Revel M. Assessment of the test-retest reliability and construct validity of a modified Lequesne index in knee osteoarthritis. Joint Bone Spine. 2003;70(6):521-5.

8. Lequesne MG, Méry C, Samson M, Marty M. Comparison between the WOMAC and the Lequesne indices in patients with knee and hip osteoarthritis. Osteoarthritis Cartilage. 1998;6(6):441-2.

9. Bellamy N, Buchanan WW, Goldsmith CH, Campbell J, Stitt LW. Validation study of WOMAC: a health status instrument for measuring clinically important patient relevant outcomes to antirheumatic drug therapy in patients with osteoarthritis of the hip or knee. J Rheumatol. 1988;15(12):1833-40.

10. Lequesne MG. The algofunctional indices for hip and knee osteoarthritis. J Rheumatol. 1997;24(4):779-81.

11. Fernandes MI. Tradução e validação do questionário de qualidade de vida específico para osteoartrose WOMAC (Western Ontario McMaster Universities) para a língua portuguesa [Dissertation]. São Paulo: Escola Paulista de Medicina, Universidade Federal de São Paulo; 2003.

12. Bijlsma JW, Knahr K. Strategies for the prevention and management of osteoarthritis of the hip and knee. Best Pract Res Clin Rheumatol. 2007;21(1):59-76.

13. Eccles M, Freemantle N, Mason J. North of England evidence-based guideline development project: summary guideline for non-steroidal antiinflammatory drugs versus basic analgesia in treating the pain of degenerative arthritis. The North of England Non-Steroidal Anti-Inflammatory Drug Guideline Development Group. BMJ. 1998;317(7157):526-30.

14. Wieland HA, Michaelis M, Kirschbaum BJ, Rudolphi KA. Osteoarthritis: an untreatable disease? Nat Rev Drug Discov. 2005;4(4):331-44.

15. Rashad S, Revell P, Hemingway A, Low F, Rainsford K, Walker F. Effect of non-steroidal anti-inflammatory drugs on the course of osteoarthritis. Lancet. 1989;2(8662):519-22. 
16. Adams ME, Lussier AJ, Peyron JG. A risk-benefit assessment of injections of hyaluronan and its derivatives in the treatment of osteoarthritis of the knee. Drug Saf. 2000;23(2):115-30.

17. Eisenberg DM, Davis RB, Ettner SL, Appel S, Wilkey S, Van Rompay M, et al. Trends in alternative medicine use in the United States, 19901997: results of a follow-up national survey. JAMA. 1998;280(18):156975.

18. Rao JK, Mihaliak K, Kroenke K, Bradley J, Tiemey WM, Weinberger M. Use of complementary therapies for arthritis among patients of rheumatologists. Ann Intern Med. 1999;131(6):409-16.

19. Astin JA. Why patients use alternative medicine: results of a national study. JAMA. 1998;279(19):1548-53.

20. Bellamy N, Buchanan WW. Outcome measurement in osteoarthritis clinical trials: the case for standardisation. Clin Rheumatol. 1984;3(3):293-303.

21. Lequesne M, Brandt K, Bellamy N, Moskowitz R, Menkes CJ, Pelletier JP, et al. Guidelines for testing slow acting drugs in osteoarthritis. J Rheumatol Suppl. 1994;41:65-71.

22. Bjordal JM, Ljunggren AE, Klovning A, Slordal L. Non-steroidal anti-inflammatory drugs, including cyclo-oxygenase-2 inhibitors, in osteoarthritic knee pain: meta-analysis of randomised placebo controlled trials. BMJ. 2004;329(7478):1317-22.

23. Anderson JW, Nicolosi RJ, Borzelleca JF. Glucosamine effects in humans: a review of effects on glucose metabolism, side effects, safety considerations and efficacy. Food Chem Toxicol. 2005;43(2):187-201.

24. Henrotin Y, Mobasheri A, Marty M. Is there any scientific evidence for the use of glucosamine in the management of human osteoarthritis? Arthritis Res Ther. 2012;14(1):201.

25. Zhang W, Doherty M, Arden N, Bannwarth B, Bijlsma J, Gunther KP, et al. EULAR evidence based recommendations for the management of hip osteoarthritis: report of a task force of the EULAR Standing Committee for International Clinical Studies Including Therapeutics (ESCISIT). Ann Rheum Dis. 2005;64(5):669-81.

26. Zhang W, Moskowitz RW, Nuki G, Abramson S, Altman RD, Arden N, et al. OARSI recommendations for the management of hip and knee osteoarthritis, part l: critical appraisal of existing treatment guidelines and systematic review of current research evidence. Osteoarthritis Cartilage. 2007;15(9):981-1000.

27. Zhang W, Moskowitz RW, Nuki G, Abramson S, Altman RD, Arden N, et al. OARSI recommendations for the management of hip and knee osteoarthritis, part II: OARSI evidence-based, expert consensus guideli- nes. Osteoarthritis Cartilage. 2008;16(2):137-62.

28. Zhang W, Nuki G, Moskowitz RW, Abramson S, Altman RD, Arden NK, et al. OARSI recommendations for the management of hip and knee osteoarthritis: part III: changes in evidence following systematic cumulative update of research published through January 2009. Osteoarthritis Cartilage. 2010;18(4):476-99.

29. Ebell MH, Siwek J, Weiss BD, Woolf SH, Susman J, Ewigman B, et al. Strength of recommendation taxonomy (SORT): a patient-centered approach to grading evidence in the medical literature. Am Fam Physician. 2004;69(3):548-56.

30. Wandel S, Jüni P, Tendal B, Nüesch E, Villiger PM, Welton NJ, et al. Effects of glucosamine, chondroitin, or placebo in patients with osteoarthritis of hip or knee: network meta-analysis. BMJ. 2010;341:e4675.

31. Towheed TE, Maxwell L, Anastassiades TP, Shea B, Houpt J, Welch V, et al. Glucosamine therapy for treating osteoarthritis. Cochrane Database Syst Rev. 2009;(2):CD002946.

32. Sawitzke AD, Shi H, Finco MF, Dunlop DD, Harris CL, Singer NG, et al. Clinical efficacy and safety of glucosamine, chondroitin sulphate, their combination, celecoxib or placebo taken to treat osteoarthritis of the knee: 2-year results from GAIT. Ann Rheum Dis. 2010;69(8):1459-64.

33. Hochberg MC, Altman RD, April KT, Benkhalti M, Guyatt G, McGowan J, et al. American College of Rheumatology 2012 recommendations for the use of nonpharmacologic and pharmacologic therapies in osteoarthritis of the hand, hip, and knee. Arthritis Care Res. 2012;64(4):465-74.

\section{CONFLITOS DE INTERESSES}

Os autores declaram não ter conflito de interesses.

\section{FINACIAMENTO}

O trabalho relatado neste manuscrito não foi objeto de qualquer tipo de financiamento externo.

\section{ENDEREÇO PARA CORRESPONDÊNCIA}

Liliana Ferreira

Travessa Moinhos, nº 35, 4410-140 São Félix da Marinha

E-mail: liliana.almeida.ferreira@gmail.com

\section{Recebido em 01-04-2014}

Aceite para publicação em 04-11-2014 


\section{ABSTRACT}

\section{GLUCOSAMINE FOR RELIEF OF SYMPTOMS OF OSTEOARTHRITIS: AN EVIDENCE-BASED REVIEW}

Objective: Osteoarthritis (OA) is a major cause of pain and disability in the elderly. Glucosamine has attracted interest as a drug for pain relief or improvement of joint function in OA. It appears to be safe. However, many studies have shown different levels of effectiveness, so its use remains controversial. The aim of this study was to review the evidence on the efficacy and safety of glucosamine in the relief of symptoms of OA.

Data Sources: Cochrane Library, PubMed, National Guideline Clearinghouse, DARE, TRIP Database, Bandolier, Index of Portuguese Medical Journals.

Methods: Meta-analyses (MA), systematic reviews, randomized controlled trials (RCT) and clinical guidelines (CG) published between 01/2009 and 12/2012 were selected, using the MeSH terms osteoarthritis and glucosamine and corresponding DeCS (Portuguese search terms). We used the Strength of Recommendation Taxonomy (SORT) scale of American Family Physician to assign levels of evidence and strength of recommendations.

Results: From the 200 articles obtained, four met the inclusion criteria, including two MA, one RCT and one CG, representing 9428 patients studied. Three articles showed that use of glucosamine had some benefit in relieving pain and improving joint function, but this benefit is not statistically or clinically significant compared to placebo. Glucosamine was found to be as safe as placebo. The CG does not recommend the use of glucosamine in OA.

Conclusion: The current available evidence suggests that benefits of glucosamine are not clinically significant in the relief of symptoms of OA, particularly in relieving pain and improving joint function (SOR A). However, it is safe (SOR A). Future studies evaluating the cost/benefit ratio of glucosamine for symptom relief may encourage or discourage its use, particularly with copayment for treatment by the National Health Service.

Keywords: Glucosamine; Osteoarthritis; Arthralgia. 